\title{
O PAPEL DAS ANIDRASES CARBONICAS NA CONSOLIDAÇÃO DA MEMÓRIA DE EXTINÇÃO
}

Eduarda_Godfried_Nachtigall; PUCRS; eduarda.godfried@gmail.com

Scheila_Daiane_Schmidt; PUCRS; scheiladschmidt@hotmail.com

Jociane_de_Carvalho_Myskiw; PUCRS; jociane_carvalho@hotmail.com

Cristiane_Regina_Guerino_Furini; PUCRS; cristianefurini@hotmail.com

Maria_Beatrice_Passani; Università degli Studi di Firenze; beatrice.passa@unifi.it

Patrizio_Blandina; Università degli Studi di Firenze; patrizio.blandina@unifi.it

Gustavo_Provensi; Università degli Studi di Firenze; gustavo.provensi@unifi.it Ivan_Izquierdo; PUCRS; ivan.izquierdo@pucrs.br

\section{RESUMO}

Introdução: A extinção de memórias forma a base da terapia de exposição, o tratamento padrão para fobias, ansiedade e transtorno de estresse pós-traumático. A extinção não apaga a memória original, mas é um novo aprendizado que inibe sua expressão. Drogas que facilitem a extinção podem representar uma nova estratégia de tratamento para diversos transtornos. As anidrases carbônicas (AC) constituem um importante grupo de enzimas envolvidas em diversos processos fisiológicos, incluindo a formação da memória. Objetivo: Investigar a participação das AC cerebrais na extinção da memória de medo condicionado ao contexto (MCC). Métodos: Ratos Wistar adultos com cânulas implantadas estereotaxicamente nas regiões CA1 do hipocampo dorsal, amígdala basolateral (BLA), córtex pré-frontal ventromedial (vmPFC) ou substância nigra (SN) foram submetidos à tarefa de MCC, na qual os mesmos receberam 3 estímulos elétricos de $0,5 \mathrm{~mA}$ nas patas. Vinte e quatro horas depois eles foram submetidos a sessão de extinção durante $15 \mathrm{~min}$ ou $30 \mathrm{~min}$. Imediatamente após, os animais receberam infusões de moduladores das AC nas regiões cerebrais alvo e, no dia seguinte, foram submetidos ao teste de retenção ( $3 \mathrm{~min}$ ). Resultados: Acetazolamida prejudicou a consolidação da memória de extinção nas regiões CA1, BLA e vmPFC, enquanto que a D-fenilalanina potencializou esse processo significativamente. Nenhum efeito foi observado quando ACTZ ou D-Phen foram infundidos na SN. Conclusão: O envolvimento das AC em regiões cerebrais fundamentais para a formação da memória é essencial para garantir a consolidação da extinção da memória de medo.

Palavras-chave: Memória de medo; Extinção; Anidrases carbônicas. 